\title{
ALFABETIZACIÓN EN SALUD EN LA COMUNIDAD
}

\section{HEALTH LITERACY IN THE COMMUNITY}

\author{
Dolors Juvinyà-Canal ${ }^{1, a}$ \\ ${ }^{1}$ Universitat de Girona, España \\ a dolors.juvinya@udg.edu
}

Recibido: 20/09/2021; Aceptado: 06/10/2021

\section{Resumen}

Este articulo trata sobre la alfabetización en salud en la comunidad. En primer lugar se clarifica que se entiende la salud como un derecho de todos los seres humanos y tiene que ser sostenida por la sociedad. Se introduce la promoción de la salud como enfoque teniendo en cuenta que se define como un proceso que permite a las personas incrementar el control sobre su salud y la importancia de la alfabetización en salud como uno de los pilares básicos.

Se desarrolla el concepto de alfabetización en salud a partir de múltiples definiciones, se revisan algunas por su significación y se recogen tres elementos claves a partir del análisis de la mayoría de ellas. Se reconoce que el concepto es multidimensional, complejo y heterogéneo. Se recopilan los conocimientos sobre el tema a partir de los estudios realizados.

Se presentan algunos de los instrumentos para la medida de la alfabetización en salud.

Por su relevancia en el contexto europeo se presenta el modelo de Sorensen y su desarrollo en el cuestionario European Health Literacy Survey que permitió conocer el nivel de alfabetización en salud en ocho países europeos.

Finalmente se introducen los entornos saludables como estrategia para facilitar la alfabetización en salud de las personas en la comunidad y se comentan los aspectos más relevantes de cada uno de ellos.

Palabras clave: Alfabetización en salud; Promoción de la Salud; Participación de la Comunidad; Entornos saludables.

\begin{abstract}
The article addresses health literacy in the community. Firstly, it clarifies that health is understood as a right of all human beings and has to be sustained by society. It introduces health promotion as an approach taking into account that it is defined as a process that allows people to increase control over their health and the importance of health literacy as one of the basic pillars.

It develops the concept of health literacy from multiple definitions, some are reviewed for their significance and three key elements are collected from the analysis of most of the definitions. The concept is recognized as multidimensional, complex, and heterogeneous. Knowledge on the subject is collected from the studies carried out.

Some of the instruments for measuring health literacy are presented.

Due to its relevance in the European context, Sorensen's model and its development are presented in the European Health Literacy Survey questionnaire that allowed us to know the level of health literacy in eight European countries.

Finally, healthy environments are introduced as a strategy to facilitate health literacy for people in the community and the most relevant aspects of each of them are discussed.
\end{abstract}


Keywords: Health Literacy; Health Promotion; Community Participation; Heathy Settings.

\section{INTRODUCCIÓN}

Antes de hablar de alfabetización en salud, consideramos importante clarificar unos aspectos previos: qué entendemos por salud, cuáles son sus determinantes y como promoverla.

La salud, es un derecho de todos los seres humanos y tiene que ser sostenida por la sociedad, tal y como está recogida en la declaración de derechos humanos. En la Carta de Ottawa la Organización Mundial de la Salud (1986), se define la Promoción de la Salud como un proceso que permite a las personas incrementar el control sobre su salud para mejorarla y que constituye un proceso político y social que engloba acciones para fortalecer las habilidades y las capacidades de las personas y las acciones para modificar las condiciones sociales, ambientales y económicas con el objetivo de disminuir las desigualdades en salud y mejorar la salud (Organización Mundial de la Salud, 1986).

Esta definición incorpora la acción comunitaria y las habilidades personales como aspectos clave para mejorar la salud de las personas. La Organización Mundial de la Salud (OMS) define el fortalecimiento de la acción comunitaria como una acción basada en el empoderamiento de la comunidad y el reforzamiento de la participación comunitaria. Concreta que la acción comunitaria para la salud hace referencia a los esfuerzos colectivos de las comunidades para incrementar el control sobre los determinantes sociales de la salud para mejorarla (Organización Mundial de la Salud, 1998).

La Carta de Ottawa también plantea que el desarrollo de aptitudes personales se enmarca en un aprendizaje a lo largo de la vida y en los diferentes entornos donde las personas desarrollan su vida. Se afirma que la promoción de la salud ha de fomentar el desarrollo personal y social de las personas proporcionando información y educación para a la salud enfocadas a mejorar las capacidades y así aumentar las opciones para mejorar el control de la propia salud. Es precisamente desde esta perspectiva donde tiene relevancia la alfabetización para la salud.

Figura 1. Los 3 pilares de la Promoción de la Salud.

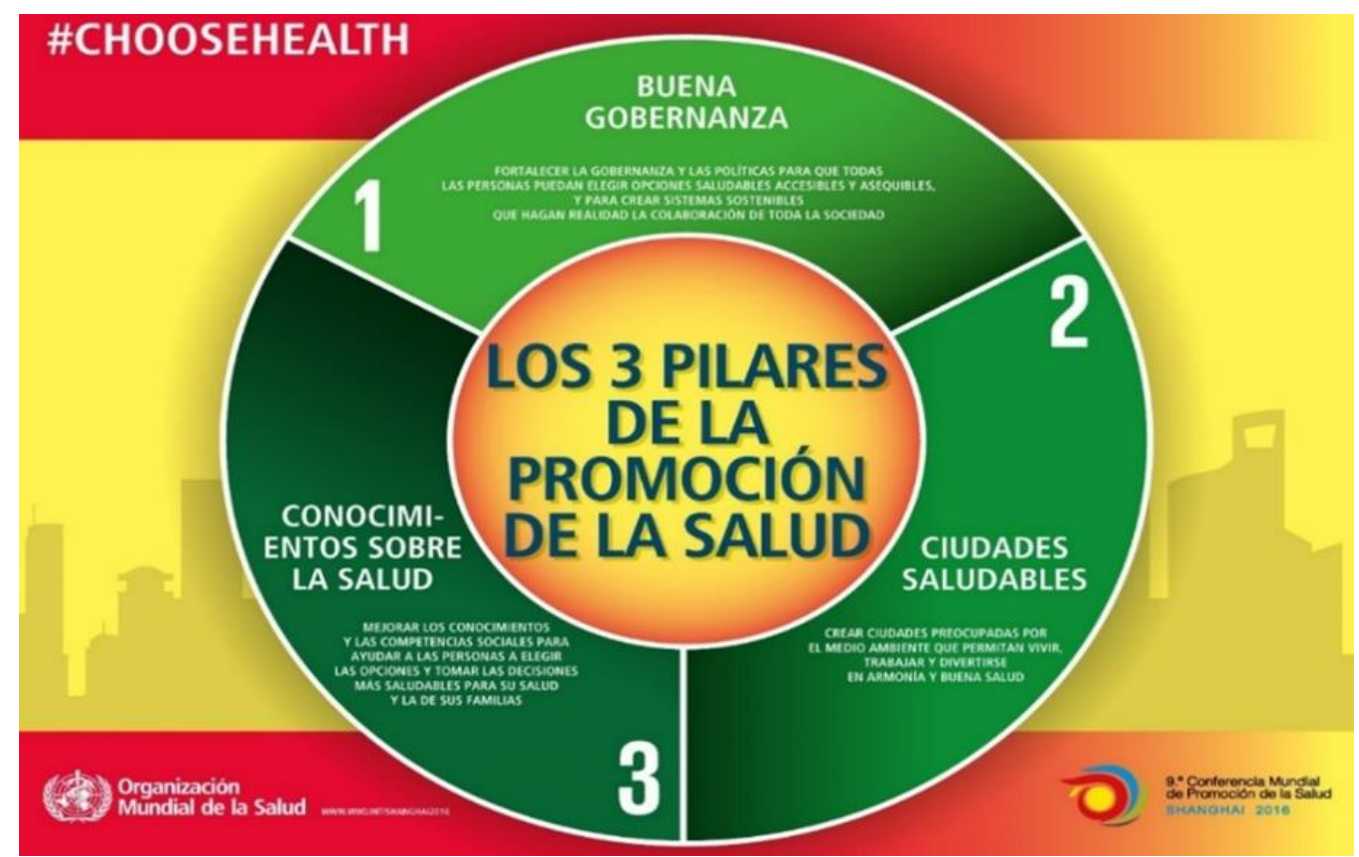

Fuente: Organización Mundial de la Salud (2016). 
En la última conferencia mundial de promoción de la salud, celebrada en Shanghái en 2016, se reconoció la alfabetización en salud como uno de los tres retos vigentes para a mejorar la salud de la población, empoderar a las personas y contribuir a la equidad (Figura 1). Además, en la declaración resultante de esta conferencia se reconoció que los conocimientos de salud (traducción que se hizo de Health Literacy) son un determinante fundamental de la salud y por tanto es necesario invertir en acciones para a mejorarlos.

Todos estos aspectos se ven recogidos y reforzados en los Objetivos de Desarrollo Sostenible (ODS) establecidos por la Organización de las Naciones Unidas en su Agenda 2030 que tienen como objetivo lograr un verdadero desarrollo sostenible a nivel mundial a través de 17 áreas de acción, algunas de las cuáles mantienen una relación directa con la salud de las personas: cambio climático, pobreza, desigualdad, educación...

\section{LA ALFABETIZACIÓN EN SALUD}

La alfabetización en salud, traducción del inglés "health literacy", fue descrita por primera vez en 1974 por Simonds en relación con la educación para la salud en la escuela. Se han formulado muchas definiciones de alfabetización en salud siendo la primera definición clara la realizada por el Comité Mixto sobre las normas de Educación sanitaria en 1995 (Sorensen et al., 2012), que establece que la Alfabetización en salud es la capacidad de las personas para obtener, interpretar y comprender la información, los servicios sanitarios y la competencia para utilizar dicha información.

La Organización Mundial de la Salud en 1998 definió la alfabetización en salud como "las habilidades cognitivas y sociales que determinan la motivación y la capacidad de las personas para acceder, comprender y utilizar la información de forma que promueva y mantenga una buena salud" (Organización Mundial de la Salud, 1986).

Kickbusch et al. propusieron en 2005 la definición de alfabetización en salud como la capacidad de tomar decisiones sanitarias acertadas en el contexto de la vida cotidiana. Es decir, en los espacios donde las personas viven y se relacionan desde la casa, en la comunidad, en el lugar de trabajo, en el sistema sanitario, en el mercado y el ámbito político. Se trata de una estrategia critica de empoderamiento para aumentar el control de las personas sobre su salud, la capacidad para buscar información y su capacidad para tomar responsabilidad (Kickbusch et al., 2005).

En las últimas décadas las definiciones han ido evolucionando y destacamos la descrita en 2012 por Sørensen et al., que la definió como un concepto estrechamente relacionado con la alfabetización que conlleva conocimiento, motivación y competencias para acceder, entender, valorar y aplicar información para tener criterio y tomar decisiones sobre el cuidado de la salud, la prevención de enfermedades y la promoción de la salud en el día a día para mantener y promover la calidad de vida a lo largo de la vida (Sorensen et al., 2012).

En 2012 también se presentó un modelo integrado de alfabetización en salud (Sorensen et al., 2012) que puede verse en la Figura 2. Este modelo consiste en la combinación de un modelo conceptual que describe las principales dimensiones de la alfabetización y que se representa con la figura ovalada del modelo, con un modelo lógico que muestra los factores proximales y distales que influyen en la alfabetización y las vías que vinculan la alfabetización con los resultados en salud. 
Figura 2: Model conceptual de HLS-EU.

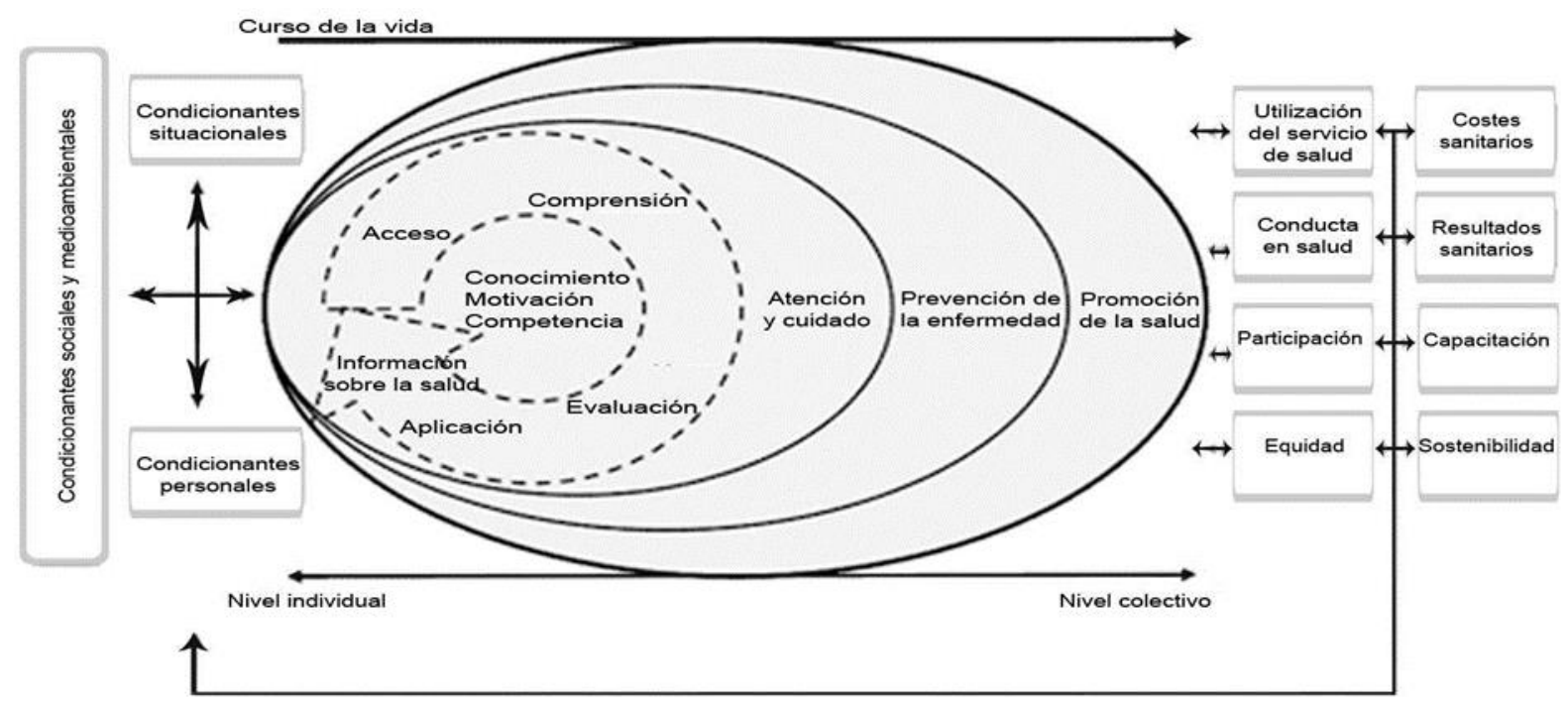

Fuente: Sorensen et al. (2012).

Se han realizado diversas revisiones sistemáticas, la de Chenxi Liu et al., recopiló 34 definiciones identificando tres elementos claves (Liu et al., 2020):

- Conocimiento de la salud, la atención sanitaria y el sistema de salud.

- Hasta qué punto las personas pueden procesar y utilizar información en diversos formatos en relación con la salud y la atención sanitaria.

- Capacidad para mantener la salud mediante el autocuidado y el trabajo en colaboración con los profesionales sanitarios.

La mayoría de investigaciones sobre alfabetización en salud la han considerado como un elemento unidimensional centrado principalmente en la capacidad de lectura o la alfabetización sanitaria funcional. Sin embargo, con la evolución del campo queda claro que la alfabetización en salud abarca múltiples dimensiones, es complejo y heterogéneo (Sorensen, 2019).

Nutbean afirmó que la educación sanitaria y la iniciativa de educación son clave para mejorar la alfabetización en salud. Describió tres tipos: La alfabetización funcional se refiere a las habilidades básicas para leer y escribir; La alfabetización interactiva engloba habilidades de alfabetización cognitivas avanzadas, que permiten una participación activa en la asistencia sanitaria; La alfabetización crítica requiere la habilidad más avanzada cognitivamente, para analizar y utilizar la información y poder participar en acciones favorables para la salud. La alfabetización interactiva y crítica proponen la expansión de la alfabetización en salud en el ámbito de la promoción de la salud a través de la su conexión con el concepto de autoeficacia y empoderamiento (Nutbean, 2000).

\section{QUÉ SE SABE DE LA ALFABETIZACIÓN EN SALUD}

Las investigaciones realizadas han aportado información relevante sobre los efectos de la alfabetización. Información útil para los profesionales y que la OMS recopiló en un monográfico como evidencia (Organización Mundial de la Salud, 2014) y señalamos a continuación. 
Se sabe que los grupos con una alta tasa de alfabetización beneficia a las sociedades ya que las personas alfabetizadas intervienen más activamente en las actividades comunitarias, están más informadas, presentan mayores ingresos y mejores trabajos.

Una limitada alfabetización en salud afecta significativamente a la salud al ir asociada a una menor participación en actividades relacionadas con el fomento de la salud y detección de enfermedades, riesgo para la salud, mayor índice de accidentes, cumplimiento de tratamientos. Obedece a un gradiente social y puede acentuar las desigualdades. Las personas con limitada alfabetización en salud muestran a menudo bajos niveles de educación por lo que no se puede separar los efectos que sobre la salud de las personas proceden de una mala alfabetización en salud.

El desarrollo de las propias capacidades y aptitudes es un proceso que dura toda la vida y la capacidad y la aptitud para la alfabetización en salud varían en función del contexto, cultura y marco dependiendo de factores personales y del sistema.

La alfabetización en salud es un capital para individuos y comunidades, fortaleciendo la alfabetización en salud hay una mejora individual y comunitaria ya que las personas adquieren y desarrollan su personal alfabetización en función de los entornos donde viven y la acción social puede mejorarlos. Es una forma de capital social ya que las comunidades se benefician de la alfabetización en salud de sus miembros y estos se benefician de apoyo y recursos comunitarios.

La alfabetización en salud significa empoderamiento ya que su objetivo es capacitar a las personas como ciudadanos, trabajadores, consumidores, pacientes... y poder tomar mejores decisiones en temas de salud y mejorar las aptitudes propias.

La competencia de la persona alfabetizada en temas de salud es capaz de buscar y evaluar la información sanitaria necesaria (Organización Mundial de la Salud, 2014):

- para comprender y seguir las instrucciones para cuidarse a sí mismo, incluyendo la aplicación de tratamientos diarios.

- para preparar y llevar a término los necesarios en el estilo de vida para mejorar la salud.

- para tomar las decisiones adecuadas relacionadas con la salud.

- para saber cuándo y dónde debe acceder al sistema sanitario en caso de necesidad y,

- para compartir actividades abordar cuestiones de salud en la comunidad y sociedad.

\section{INSTRUMENTOS PARA LA MEDIDA DE LA ALFABETIZACIÓN EN}

\section{SALUD}

Son múltiples los instrumentos disponibles para medir el nivel de alfabetización en salud. Algunos utilizados principalmente en el ámbito de la salud y otros a nivel comunitario. A continuación, destaremos algunos de los más utilizados (Gonzalez y Serra, 2014).

El Test of Functional Health Literacy in Adults (TOFHLA) diseñado por Parker, mide la alfabetización en salud funcional, valorando la habilidad de la persona para leer textos y frases que incluyen cifras, utilizando material del ámbito sanitario. El tiempo de realización es de 22 minutos, pero presenta una fiabilidad excelente y una validez buena.

La escala Rapid Estimate of Adult Literacy in Medicine (REALM) elaborada por Davis et al., detecta el nivel de alfabetizaciones para la salud de pacientes en el ámbito de atención primaria, 
educación sanitaria e investigación médica. Es un test de reconocimiento de 125 palabras, donde el paciente debe leer e identificar las palabras relacionadas con la salud y clasificarlas en niveles. Es un test rápido y con alta fiabilidad.

El Medical Achievement Reading Test (MART) de Hanson-Divers, es un test funcional que se diseñó para personas con dificultades para leer. Contiene 42 palabras, entre ellas vocabulario médico, etiquetas de fármacos, recetas y envases que deslumbran y dificultan la lectura. Las ventajas son la rapidez y la alta fiabilidad.

El Short Assessment of Health Literacy for Spanish-speaking Adults (SAHLSA) de Lee et al., es un test de reconocimiento de palabras con un test de comprensión para la población hispana. El test valora la comprensión y la pronunciación. Es rápido, fácil de realizar y tiene una elevada fiabilidad, aunque la validez es limitada.

La Health Activities Literacy Scale (HALS) es una escala funcional, que estudia las Habilidades de alfabetización relacionadas con la salud. Consta de 191 ítems y está dirigida a la población adulta. Este test clasifica las competencias relacionadas con la salud de cinco dominios: promoción de la salud, protección de la salud, prevención de enfermedades, cuidados y mantenimiento de la salud y sistemas de navegación. Algunos investigadores consideran que es la mejor herramienta para medir la alfabetización para la salud (Organización Mundial de la Salud, 2009).

La eHealth Literacy Scale (Health) de Norman y Skinner, es una escala para medir de forma rápida y sencilla la competencia de eSalud. Consta de 8 ítems y evalúa conocimientos, confort y habilidades en el proceso de búsqueda, evaluación y aplicación de información electrónica relacionada con problemas de salud. Es una herramienta basada en la percepción subjetiva que tienen los usuarios sobre las habilidades y conocimientos que tienen sobre la salud digital.

El cuestionario European Health Literacy Survey (HLS-EU) de Sorensen et al., fue formado a partir del modelo conceptual de la alfabetización para la salud descrito anteriormente. Consta de 47 preguntas con 4 tipos de respuesta (muy fácil, fácil, difícil o muy difícil). Estas preguntas se centran en determinadas situaciones donde se necesita gestionar información para la salud en aspectos relacionados con la asistencia sanitaria, la prevención de enfermedades y la promoción de la salud (Sorensen et al., 2012).

El Health Literacy Questionaire (HLQ) de Osborne et al. (2013) es una herramienta multidimensional que diagnostica las necesidades de alfabetización en salud entre personas y organizaciones. Tiene 44 preguntas y estudia 9 dimensiones, las opciones de respuesta son de cuatro puntos de muy en desacuerdo en muy de acuerdo (Osborne et al., 2013).

\section{LA ALFABETIZACIÓN PARA LA SALUD EN EUROPA}

En 2011 se inició el proyecto European Health Literacy Survey (HLS-EU) para estudiar la alfabetización en salud de la población y sus determinantes, y establecer una red europea de alfabetización para la salud. En este proyecto participaron 8 países entre los que se encuentra España. Los objetivos del proyecto eran: tener un instrumento para medir la alfabetización en salud en Europa, cuantificar los datos de alfabetización en salud en los diferentes países europeos y crear órganos consultivos nacionales.

La Encuesta Europea sobre Alfabetización en Salud puso de manifiesto que, en Europa, casi la mitad $(47,6 \%)$ de las personas encuestadas tenían una alfabetización en salud limitada (problemática o inadecuada), En la figura 3 se muestran los porcentajes de los diferentes niveles de alfabetización en los 8 países participantes. Un 12,4\% de los europeos presentan una 
alfabetización para la salud inadecuada y un 35,2 alfabetización problemática. Es decir, una de cada dos personas en Europa presentan una alfabetización en salud limitada.

Los resultados de la muestra española presentan un porcentaje por debajo de la media europea en alfabetización en salud inadecuada $(7,5 \%)$ pero presenta un elevado porcentaje en alfabetización problemática (50,8\%).

Figura 3: Nivel general de alfabetización en salud en Europa.

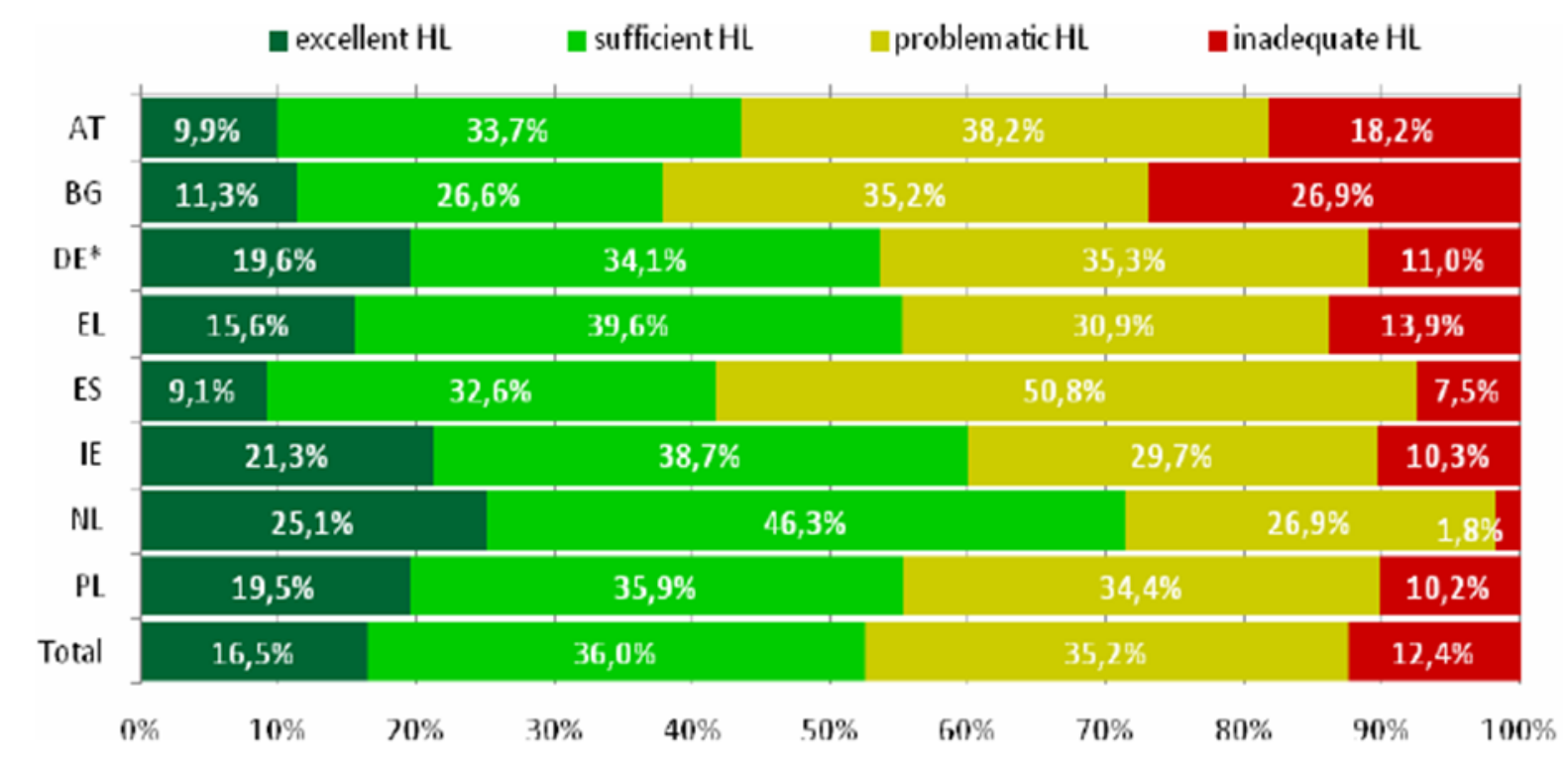

Fuente: Sorensen et al. (2012).

En un estudio que se hizo en 2014 en Cataluña, incorporando el cuestionario HLS-EU en la Encuesta de salud de Cataluña los resultados mostraron un porcentaje de población con un nivel de alfabetización en salud limitado del 15,4\% y menor entre los estudiantes universitarios $(8,3 \%)$ (Garcia-Codina et al., 2019). Se observó también que la edad es inversamente proporcional a la alfabetización en salud.

\section{ENTORNOS FAVORABLES PARA LA ALFABETIZACIÓN EN SALUD}

Ya en la Carta de Ottawa en 1986, una de las acciones que propone para mejorar la salud es la de creación de ambientes favorables a la salud. Posteriormente en la tercera conferencia internacional de promoción de la salud en Sundswal en 1991 se centró en el concepto y la práctica de creación de ambientes saludables, y en factores físicos y sociales que promueven la salud donde las personas viven, estudian, trabajan y disfrutan. Poniendo de manifiesto que es importante identificarlos para desarrollar intervenciones en estos espacios (OMS, 2012)

Posteriormente la Organización Mundial de la Salud (OMS) impulso la estrategia de entornos saludables para promover la salud de las personas promoviendo distintos programas que han dado origen a su vez a diferentes redes nacionales e internacionales. El primero de ellos fue el de Ciudades, posteriormente Escuelas, Hospitales, Prisiones, centros de trabajo, universidades entre otros.

El enfoque de entornos implica un método holístico e interdisciplinario reconociendo la importancia de los contextos. Los elementos clave son la participación de la comunidad, la cooperación, el empoderamiento y la equidad siendo la alfabetización en salud una parte 
esencial.

A continuación, comentamos algunos aspectos relevantes de estos entornos.

La ciudad. El movimiento ciudades saludables ha sido un elemento clave reconociendo la importancia de la salud, promoviendo entornos físicos y sociales y desarrollando recursos comunitarios que favorezca que las personas desarrollen sus actividades vitales. Su implementación requiere un liderazgo comprometido con la salud, un compromiso político y una cooperación intersectorial y de la participación de las personas en la toma de decisiones.

La alfabetización para la salud tiene un papel importante para lograr los objetivos en los dos campos de acción el social y colectivo, creando un medio ambiente y entornos favorables al desarrollo de las personas y el individual, desarrollando las aptitudes personales para adoptar estilos de vida saludables.

La escuela como entorno educativo juega un papel importante para asentar las bases de alfabetización en salud desde el desarrollo de la primera infancia. Incluye también la relación con los padres y familia. Una escuela promotora de salud implica al personal de educación y el de salud, a los profesores, estudiantes, familias y líderes de la comunidad, en la tarea de promover la salud. Fomenta la salud y el aprendizaje con todos los medios a su alcance, procurando ambientes favorables para la salud y una serie de programas y servicios clave de promoción y educación para la salud. Por su importancia tiene un apartado especial en este monográfico.

El Hospital es un entorno de atención a la salud muy significativo para las personas, el proceso de curación de las enfermedades en los mismos puede realizarse de tal manera que pueden también constituirse en un referente de lo saludable y un apoyo al proceso poblacional de crear salud (OMS, 2007). El hospital es un promotor de cultura en el contexto local, y es un ejemplo para otras instituciones, por lo que las acciones que en él se desarrollen repercutirán en la comunidad. Se considera que la hospitalización puede ser una oportunidad para desarrollar actividades de promoción de la salud, que se debe implementar con otras similares realizadas en la comunidad. Dada la complejidad del sistema y a los múltiples requisitos que se enfrentan las personas es importante que los centros establezcan una política que promueva la alfabetización en salud.

El entorno laboral es un lugar o contexto social en los que las personas participan en actividades diarias, donde los factores ambientales, organizacionales y personales interactúan para favorecer la salud.

Un entorno de trabajo saludable según la Organización Mundial de la Salud es aquel en el que trabajadores y jefes colaboran en un proceso de mejora continua para promover y proteger la salud, seguridad y bienestar de los trabajadores y la sustentabilidad del ambiente de trabajo. Se incide en el espacio físico de trabajo, en el medio psicosocial del trabajo incluyendo la organización del mismo y la cultura del ambiente de trabajo. Cabe señalar que los recursos de salud personales en el espacio de trabajo incidirán en las formas en que la comunidad busca mejorar la salud de las personas, sus familias y de otros miembros de la comunidad. (Organización Mundial de la Salud, 2010).

La universidad es otro entorno que facilita promover la salud del alumnado y de los trabajadores. Permite implementar estrategias para mejorar hábitos saludables de sus miembros que trascenderán los espacios universitarios cumpliendo con uno de sus principios básico que es la vinculación con la comunidad a la que pertenece (Martinez-Riera et al., 2018). 


\section{PARA CONCLUIR}

La alfabetización en salud es multidimensional e interdisciplinaria, dando cabido a gran variedad de profesionales implicados en la mejora de la salud de las personas y las comunidades más allá de los profesionales de la salud. Precisamente por su complejidad requiere de un trabajo colaborativo que se extienda más allá de los servicios de salud, impulsando el diseño de espacios comunitarios y entornos orientados a la mejora de la alfabetización en salud de los grupos y las comunidades.

Mejorar la alfabetización en salud es necesario para reducir las inequidades en salud y para ello es necesario información buena, fiable y accesible dirigida a las necesidades y circunstancias de los distintos grupos sociales (Kickbusch et al., 2005).

De las áreas potenciales de acción destacan el desarrollar políticas que reconozcan la alfabetización en salud como un derecho ya que junto con la alfabetización forma parte de las competencias fundamentales para que las personas puedan desenvolverse en la sociedad actual.

En las iniciativas de alfabetización para la salud funcionan mejor cuando se adaptan a enfoques que contemplan las diversas formas que tienen las personas de abordar la salud. Por lo que hay que tener en cuenta el contexto social, el papel de la familia, la cultura y la educación a la hora de diseñar programes o acciones. (Organización Mundial de la Salud, 2014).

\section{REFERENCIAS BIBLIOGRÁFICAS}

Bostock, S. y Steptoe, A. (2012). Association between low functional health literacy and mortality in older adults: longitudinal cohort study. BMJ, 344(mar15 3), e1602-e1602. https://doi.org/10.1136/bmj.e1602

Garcia-Codina, O., Juvinyà-Canal, D., Amil-Bujan, P., Bertran-Noguer, C., González-Mestre, M. A., Masachs-Fatjo, E., ... Saltó-Cerezuela, E. (2019). Determinants of health literacy in the general population: results of the Catalan health survey. BMC Public Health, 19(1), 1122. https://doi.org/10.1186/s12889-019-7381-1

Gonzalez, M. y Serra M. (2014). Com es mesura l'alfabetització per a la salut? en R. Suñer y M. Santiña (Eds.), Alfabetització per a la salut. Estandars i recomanacions per als Professionals de la salut (pp. 27-44). Documenta Editorial.

Juvinyà-Canal, D., Bertran-Noguer, C. y Suñer-Soler, R. (2018). Alfabetización para la salud más que información. Gaceta Sanitaria, 32(1), 8-10. https://doi.org/10.1016/j.gaceta.2017.07.005

Kickbusch, I., Wait, S. y Maag, D. (2005). Navigating health. The role of health literacy. Alliance for Health and the Future, International Longevity Centre.

Liu, C., Wang, D., Liu, C., Jiang, J., Wang, X., Chen, H., ... Zhang, X. (2020). What is the meaning of health literacy? A systematic review and qualitative synthesis. Family Medicine and Community Health, 8(2), e000351. https://doi.org/10.1136/fmch-2020-000351

Martínez-Riera, J. R., Gallardo Pino, C., Aguiló Pons, A., Granados Mendoza, M. C., López-Gómez, J. y Arroyo Acevedo, H. V. (2018). La universidad como comunidad: universidades promotoras de salud. Informe SESPAS 2018. Gaceta Sanitaria, (32), 86-91. https://doi.org/10.1016/j.gaceta.2018.08.002

Nutbean, D. (2000). Health literacy as a public goal: A challenge for contemporary health education and communication strategies into the 21st century. Health Promotion International, 15(3), 259-267. https://doi.org/10.1093/heapro/15.3.259 
Organización Mundial de la Salud (1986). Carta de Ottawa de Promoción de la Salud. Organización Mundial de la Salud.

Organización Mundial de la Salud (1998). Glosario de Promoción de la Salud. Ministerio de Sanidad y Consumo.

Organización Mundial de la Salud (2007). La implementación de la Promoción de la salud en los hospitales. Ministerio de Sanidad y Consumo.

Organización Mundial de la Salud (2010). Entornos laborales saludables. Fundamentos y modelos. Organización Mundial de la Salud.

Organización Mundial de la Salud (2014). Alfabetización para la Salud: Datos contrastados. Documenta Universitaria.

Organización Mundial de la Salud (2016). Declaracion de Shangai. Recuperado de https://Shanghaideclaration-final-draft-es.pdf.pdf (who.int)

Osborne, R. H., Batterham, R. W., Elsworth, G. R., Hawkins, M. y Buchbinder, R. (2013). The grounded psychometric development and initial validation of the Health Literacy Questionnaire (HLQ). BMC Public Health, 13(1), 658. https://doi.org/10.1186/1471-2458$\underline{13-658}$

Sørensen, K., Van den Broucke, S., Fullam, J., Doyle, G., Pelikan, J., Slonska, Z. y Brand, H. (2012). Health literacy and public health: A systematic review and integration of definitions and models. BMC Public Health, 12(1), 80. https://doi.org/10.1186/1471-2458-12-80

Sørensen, K., Pelikan, J. M., Röthlin, F., Ganahl, K., Slonska, Z., Doyle, G., ... Brand, H. (2015). Health literacy in Europe: comparative results of the European health literacy survey (HLSEU). The European Journal of Public Health, 25(6), 1053-1058. https://doi.org/10.1093/eurpub/ckv043

Sørensen, K. (2019). Defining health literacy: Exploring differences and commonalities. En O. Okan, U. Bauer, D. Levin-Zamir, P. Pinheiro y K. Sørensen (Eds), International Handbook of health literacy: Research, practice and policy across the lifespan (pp. 5-20). Policy Press. 